\title{
Is Tip Apex Distance As Important As We Think? A Biomechanical Study Examining Optimal Lag Screw Placement
}

\author{
Patrick Kane MD, Bryan Vopat MD, Wendell Heard MD, Nikhil Thakur MD, \\ David Paller MD, Sarath Koruprolu BS, Christopher Born MD
}

Received: 1 October 2013/Accepted: 20 March 2014/Published online: 24 April 2014

(C) The Association of Bone and Joint Surgeons \& 2014

\begin{abstract}
Background Intertrochanteric hip fractures pose a significant challenge for the orthopaedic community as optimal surgical treatment continues to be debated. Currently, varus collapse with lag screw cutout is the most common mode of failure. Multiple factors contribute to cutout. From a surgical technique perspective, a tip apex distance less than $25 \mathrm{~mm}$ has been suggested to decrease the risk of cutout. We hypothesized that a low-center lag
\end{abstract}

One of the authors $(\mathrm{CB})$ certifies that he, or a member of his immediate family, has or may receive payments or benefits, during the study period, an amount of USD 10,000-USD 100,000 from Stryker Orthopaedics (Mahwah, NJ, USA); one author (CB) has stock in BioIntraface (North Kingston, RI, USA) and IlluminOss (Riverside, RI, USA).

Intramedullary nails and instrumentation equipment were provided by Stryker.

All ICMJE Conflict of Interest Forms for authors and Clinical Orthopaedics and Related Research editors and board members are on file with the publication and can be viewed on request.

Clinical Orthopaedics and Related Research neither advocates nor endorses the use of any treatment, drug, or device. Readers are encouraged to always seek additional information, including FDAapproval status, of any drug or device prior to clinical use.

Each author certifies that his or her institution approved the human protocol for this investigation, that all investigations were conducted in conformity with ethical principles of research, and that informed consent for participation in the study was obtained.

This work was performed at RIH Orthopaedic Foundation, Inc, Providence, RI, USA.

P. Kane $(\bowtie)$, B. Vopat, C. Born

Department of Orthopaedics, Rhode Island Hospital, 593 Eddy

Street, Providence, RI 02903, USA

e-mail:pmkkane@gmail.com; patrick_kane@yahoo.com

W. Heard

Department of Orthopaedic Surgery, Tulane University School of Medicine, New Orleans, LA, USA screw position in the femoral head, with a tip apex distance greater than $25 \mathrm{~mm}$ will provide equal, if not superior, biomechanical stability compared with a center-center position with a tip apex distance less than $25 \mathrm{~mm}$ in an unstable intertrochanteric hip fracture stabilized with a long cephalomedullary nail.

Questions/purposes We attempted to examine the biomechanical characteristics of intertrochanteric fractures instrumented with long cephalomedullary nails with two separate lag screw positions, center-center and low-center. Our first research purpose was to examine if there was a difference between the center-center and low-center groups in cycles to failure and failure load. Second, we analyzed if there was a difference in fracture translation between the study groups during loading.

Methods Nine matched pairs of femurs were assigned to one of two treatment groups: low-center lag screw position and center-center lag screw position. Cephalomedullary nails were placed and tip apex distance was measured. A standard unstable four-part intertrochanteric fracture was created in all samples. The femurs were loaded dynamically until failure. Cycles to failure and load and displacement data were recorded, and three-dimensional (3-D) motion was recorded using an Optotrak ${ }^{\circledR}$ motion tracking system.

Results There were no significant differences between the low-center and center-center treatment groups regarding

\author{
N. Thakur \\ Department of Orthopaedics, Upstate Bone and Joint Center, \\ Upstate Medical University-SUNY, Syracuse, NY, USA \\ D. Paller, S. Koruprolu \\ RIH Orthopaedic Foundation, Inc, Providence, RI, USA
}


the mean number of cycles to failure and mean failure load. The 3-D kinematic data showed significantly increased motion in the center-center group compared with the lowcenter group. At the time of failure, the magnitude of fracture translation was statistically significantly greater in the center-center group $(20 \pm 2.8 \mathrm{~mm})$ compared with the low-center group $(15 \pm 3.4 \mathrm{~mm} ; \mathrm{p}=0.004)$. Additionally, there was statistically significantly increased fracture gap distraction (center-center group, $13 \pm 2.8$ versus lowcenter group, $7 \pm 4 ; \mathrm{p}<0.001)$ and shear fracture gap translation (center-center group, $12 \pm 2.3 \mathrm{~mm}$; low-center group, $6 \pm 2.7 \mathrm{~mm}$; $\mathrm{p}<0.001)$.

Conclusions Positioning of the lag screw inferior in the head and neck was found to be at least as biomechanically stable as the center-center group although the tip apex distance was greater than $25 \mathrm{~mm}$.

Clinical Relevance Our findings challenge previously accepted principles of optimal lag screw placement.

\section{Introduction}

Intertrochanteric hip fractures continue to pose problems for orthopaedic surgeons. Even with improvements in the devices and techniques used to treat these fractures, failures continue to occur. The most common mode of failure is varus collapse with cutout of the lag screw in the femoral head [3, $4,8]$. Cutout rates for intramedullary nails may be as much as $8 \%$ [13] and can result in a loss of reduction, leading to pain, decreased joint mobility, leg length discrepancy, and limping [7]. Factors that lead to cutout include patient age, bone quality, fracture pattern, stability of the reduction, implant angle, and the position of the lag screw in the femoral head. However, the importance of each factor is unclear [1]. Baumgaertner et al. [1] introduced the concept of the tipapex distance as a clinically useful way to predict cutout of the screw used for proximal fixation of intertrochanteric fractures of the hip. Their landmark study was a retrospective review of 198 fractures treated with either a side-plate cephalomedullary device or an intramedullary cephalomedullary device. Their results support the center-center position with central deep placement of the screw in the femoral head such that the sum of the distance from the tip of the screw to the apex of the femoral head in the AP plane and lateral plane is less than $25 \mathrm{~mm}$ [1].

An alternative to the center-center position is the lowcenter position, in which the tip of the lag screw is placed in the lower $1 / 3$ of the femoral head on the AP intraoperative fluoroscopic view and in the center of the femoral head on the lateral view. Placement of the lag screw in this position often will lead to a tip apex distance measurement that is greater than the $25 \mathrm{~mm}$ suggested by Baumgaertner et al. [1].
In this cadaveric vertical loading study, we hypothesized that a low-center screw position with a tip apex distance greater than $25 \mathrm{~mm}$ would provide equal biomechanical properties compared with a center-center position with a tip apex distance less than $25 \mathrm{~mm}$. The specific questions we asked were: is there a difference between center-center and low-center positions regarding cycles to failure and failure load, and is there a difference in fracture translation during cycling.

\section{Materials and Methods}

To test our hypothesis, we performed a cadaveric study evaluating the biomechanical properties of an unstable intertrochanteric hip fracture model stabilized with a long cephalomedullary intramedullary nail (Stryker ${ }^{\circledR}$ Long Gamma ${ }^{\circledR} 3$ Nail; Stryker Orthopaedics, Mahwah, NJ, USA) locked with a single screw (Stryker Fully Threaded 5 x $35 \mathrm{~mm}$ Locking Screws; Stryker Orthopaedics) in the dynamic position distally and the lag screw (Stryker Lag Screw; Stryker Orthopaedics) placed in either the centercenter (tip apex distance $25 \mathrm{~mm}$ or less) or low-center (tip apex distance greater than $25 \mathrm{~mm}$ ) position.

Eighteen fresh frozen human female femurs (nine matched pairs; age, $82 \pm 3.6$ years; range, $76-89$ years) were used for this study. Fresh frozen cadaver tissue is the best medium for evaluating biomechanical properties because it most closely approximates in vivo biomechanical characteristics [17]. The specimens were screened for gross anatomic defects and excluded from the study if abnormalities were found. Bone mineral density measurements were performed on the proximal femur using dual-energy X-ray absorptiometry with a Hologic ${ }^{\circledR}$ QDR $^{\mathrm{TM}} 1000$ DEXA imager (Hologic ${ }^{\circledR}$ Inc, Marlborough, MA, USA). There was no statistical difference between the two groups regarding bone quality (mean density in the center-center group $0.63 \pm 0.24 \mathrm{~g} / \mathrm{cm}^{2}$ compared with $0.66 \pm 0.22$ $\mathrm{g} / \mathrm{cm}^{2}$ in the low-center group; $\mathrm{p}=0.222$ ).

The specimens were maintained in a freezer at $-20^{\circ} \mathrm{C}$ until approximately 12 hours before mechanical testing. The specimens were thawed to room temperature and all residual soft tissue and musculature surrounding the femur was removed by careful dissection.

\section{Sample Instrumentation}

The 18 femurs were assigned to one of two treatment groups: the center-center (right femur) or low-center (left femur) position for the lag screw. Surgeons (PK, NT, WH) trained in these treatment techniques performed all surgical procedures in accordance with the instructions provided by 
the manufacturer. Intramedullary nail and lag screw angles were measured for each femur. The proximal set screw was placed to allow sliding of the lag screw, and a single distal interlocking screw in the dynamic position was used in all instances. Fluoroscopy was used to ensure appropriate placement of the intramedullary nail, lag screw, and distal interlock screw. AP and lateral radiographs were obtained to ensure proper implant placement and to measure tip apex distance. The mean tip apex distance in the center-center group was $21 \pm 3.0 \mathrm{~mm}$ compared with $31 \pm 3.0 \mathrm{~mm}$ in the low-center group.

Custom marker flags for use with the Optotrak ${ }^{\circledR}$ system (Northern Digital, Waterloo, Ontario, Canada) were secured to the femur proximal (femoral neck) and distal (proximal femoral shaft) to the fracture site. Additional flags were oriented on the lag screw and distal femur below the distal interlock transverse screw.

\section{Creation of Fracture}

A standard, unstable four-part intertrochanteric fracture was created using a straight sagittal saw as described by Kaufer et al. [6]. A cut was made through the anterior femoral attachment of the joint capsule distally spanning the medial femoral cortex and proximally spanning the tip of the greater trochanter. A second cut was made perpendicular to the anterior capsule line passing through the abductor tubercle of the greater trochanter. A final cut was made perpendicular to the anterior cut passing the base of the lesser trochanter and femoral neck.

\section{Dynamic Testing}

The distal condyles were potted in Smooth-Cast ${ }^{\mathbb{R}}$ Urethane Series 300 potting material (Smooth- On Inc, Easton, PA, USA) with the assistance of a custom alignment fixture. The potted condyles then were mounted in a double gimbal fixture facilitating unconstrained motion in the sagittal and coronal planes. Proximally, the head of the femur was coupled with the actuator of an MTS ${ }^{\circledR} 810$ Servohydraulic Load Frame $\left(\right.$ MTS $^{\circledR}$ Corp, Eden Prairie, MN, USA) (Fig. 1). For all tests, the loading vector was oriented in the center of the femoral head passing through the intercondylar notch in the coronal plane and the femoral epicondyles in the sagittal plane. Initially, samples were loaded dynamically in compression at $700 \mathrm{~N}(1 \times$ body weight of $70 \mathrm{~kg}$ ) with a test frequency of $1 \mathrm{~Hz}$ for a total of 500 cycles $[10,16]$. On completion of 500 cycles, the loading magnitude was increased $350 \mathrm{~N}$ ( $1 \frac{1}{2} \mathrm{x}$ body weight) and retested for an additional 500 cycles. This ramping load sequence was repeated every 500 cycles until dynamic

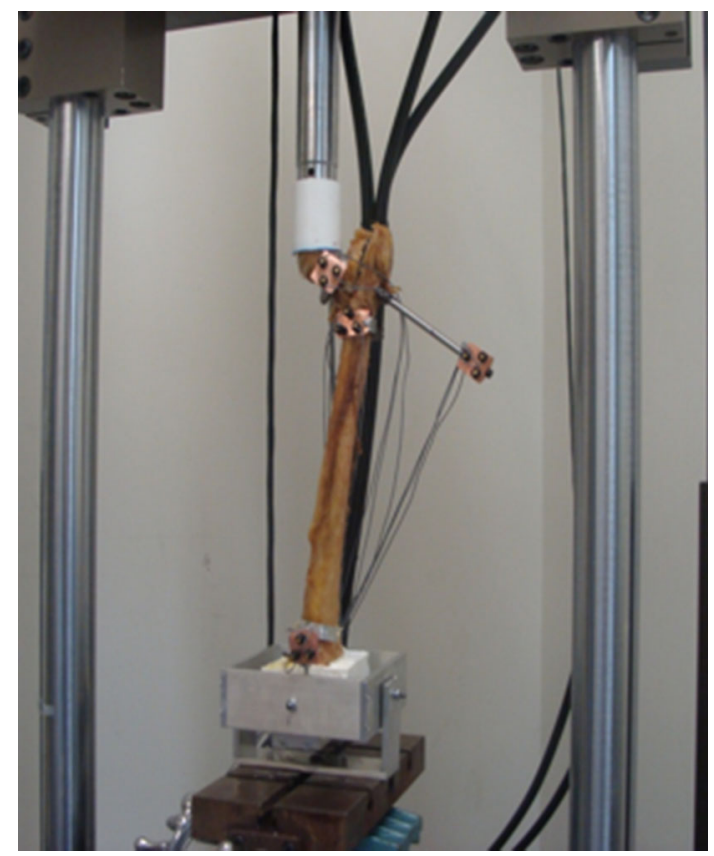

Fig. 1 The potted condyles were mounted in a double gimbal fixture facilitating unconstrained motion in the sagittal and coronal planes. The head of the femur was coupled with the actuator of an MTS ${ }^{\circledR} 810$ Servohydraulic Load Frame.

failure, reported as greater than $1.5 \mathrm{~cm}$ of height loss (plastic and elastic deformation) from the start of dynamic testing at $700 \mathrm{~N}$ [9]. Evidence of lag screw cutout and varus collapse of the construct at failure was noted during testing (Fig. 2).

Load and displacement data were recorded digitally at a frequency of $10 \mathrm{~Hz}$. The relative three-dimensional (3-D) motion of the proximal femur facture segment (femoral head), distal femur fracture segment (shaft and greater trochanter), distal femoral condyles, and lag screw were measured by an Optotrak ${ }^{\circledR} 3020$ motion tracking system (Northern Digital) and these data were synchronized with the load data from the MTS ${ }^{\circledR}$ electronics [12]. To determine global changes in motion, the relative difference in position between the rigid body segments proximal and distal to the fracture was analyzed along the applied motion axes. Namely, each femur was oriented and digitized such that components of motion between the femur segments would be identifiable. Motion perpendicular to the initial osteotomy cut of the femur was defined as fracture distraction or compression. Motion along a parallel plane to the initial osteotomy cut was defined as shear translation. The magnitude of the fracture gap transition was defined as the square root of the sum of squares of each component of motion. In all instances, translation measures were defined at a point coincidental or immediately before catastrophic failure. Optotrak ${ }^{\circledR}$ data were recorded for the initial 20 

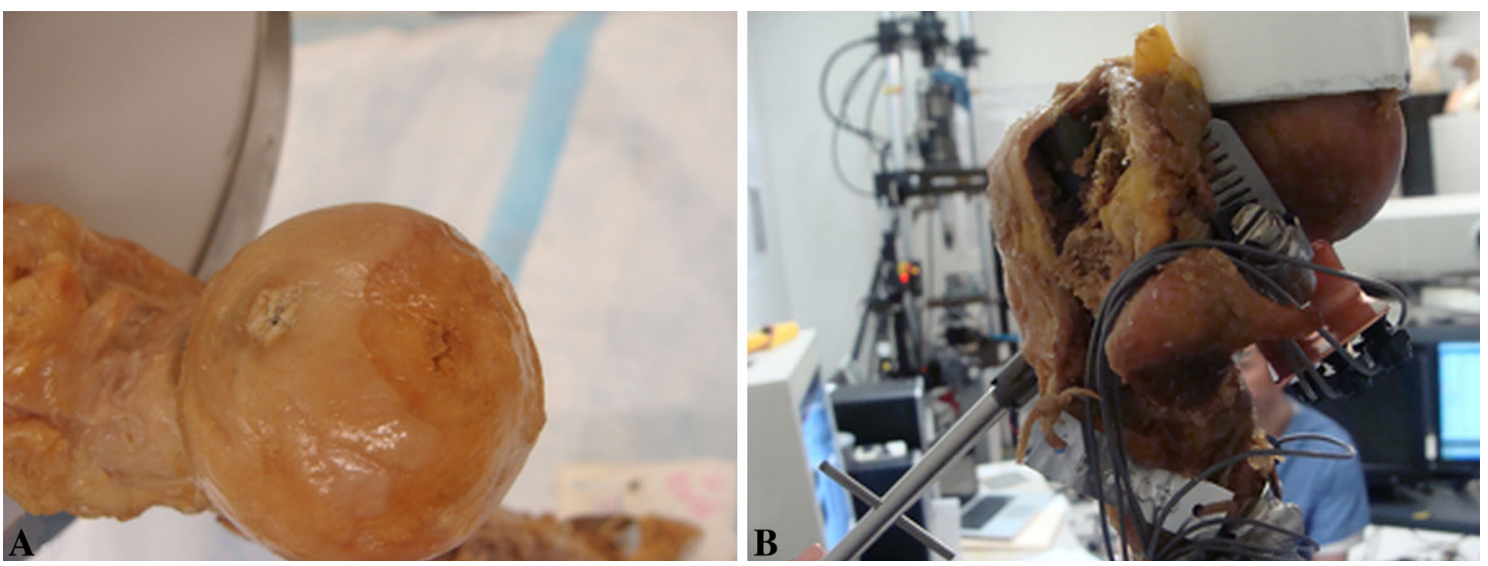

Fig. 2A-B Samples with (A) lag screw cutout at clinical failure ( $>1.5 \mathrm{~cm}$ height loss), and (B) varus collapse at clinical failure $(>1.5 \mathrm{~cm}$ height loss) are shown.

cycles at the start of mechanical testing and the initial 20 cycles for every subsequent 100-cycles.

\section{Statistical Analysis}

Kaplan-Meier survival analyses were performed using GraphPad Version 5.0 (GraphPad Software Inc, La Jolla, CA) to determine if statistically significant differences existed between the number of cycles to failure and the failure load magnitude between the center-center and lowcenter treatment groups. A Mantel-Cox log rank and GehanBreslow-Wilcoxon test were used to determine if statistical differences existed between groups for the survival analysis. Additionally, a paired Student's t-test was performed to determine if statistical differences were evident between the mean failure load and number of cycles completed at failure. Paired Student's t-tests were used to compare fracture kinematics between the two groups for shear fracture translation, fracture distraction, and the overall magnitude of fracture translation. The study was powered to detect a $20 \%$ difference in the magnitude of loading cycles or fracture translation between treatment groups. In all cases, statistical significance was set to a probability less than 0.05 a priori.

\section{Results}

Failure Load and Dynamic Survival Analysis

Kaplan-Meier survival analysis indicated there was no significant difference between the two treatment groups regarding the mean number of cycles to failure (Fig. 3A) (Mantel-Cox, $\mathrm{p}=0.925$; Gehan-Breslow-Wilcoxon test, $\mathrm{p}=0.827$ ) or mean failure load (Fig. 3B) (Mantel-Cox, $\mathrm{p}=0.911 ;$ Gehan-Breslow-Wilcoxon test, $\mathrm{p}=0.694)$.
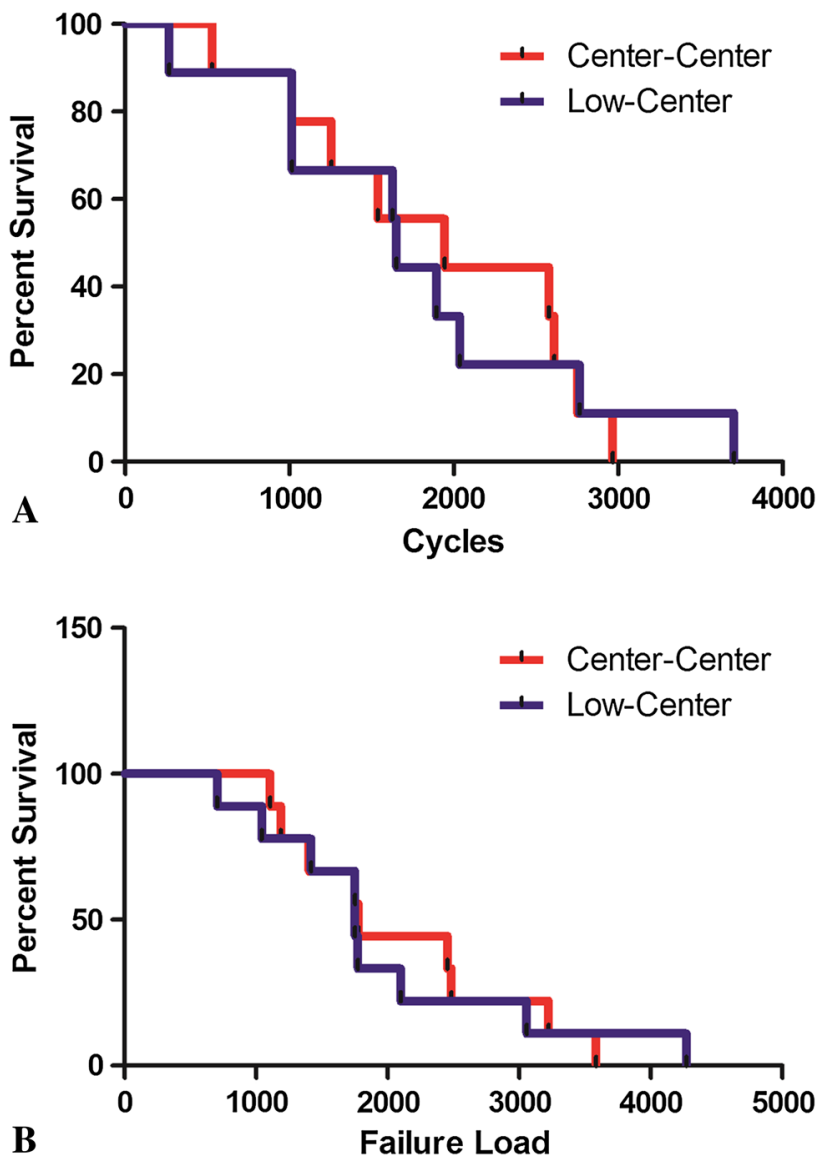

Fig. 3A-B Kaplan-Meier survival analyses indicated no significant differences between the two treatment groups for the (A) mean number of cycles to failure (Mantel-Cox, $\mathrm{p}=0.925$; Gehan-BreslowWilcoxon, $\mathrm{p}=0.827$ ) or (B) mean failure load (Mantel-Cox, $\mathrm{p}=0.911$; Gehan-Breslow-Wilcoxon, $\mathrm{p}=0.694)$.

The mean cutout failure load for samples in the low-center group was $1985 \pm 1083 \mathrm{~N}$ compared with $2108 \pm 886 \mathrm{~N}$ in the center-center group $(\mathrm{p}=0.514,1-\beta=0.05,154$ 
Mean Fracture Translation

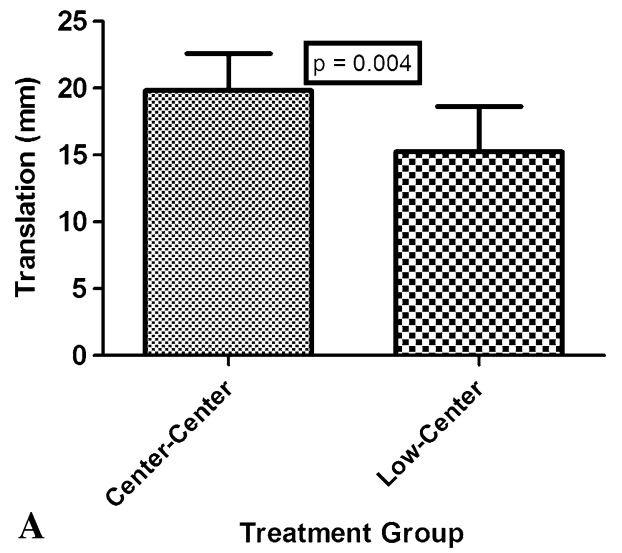

Shear Fracture Translation

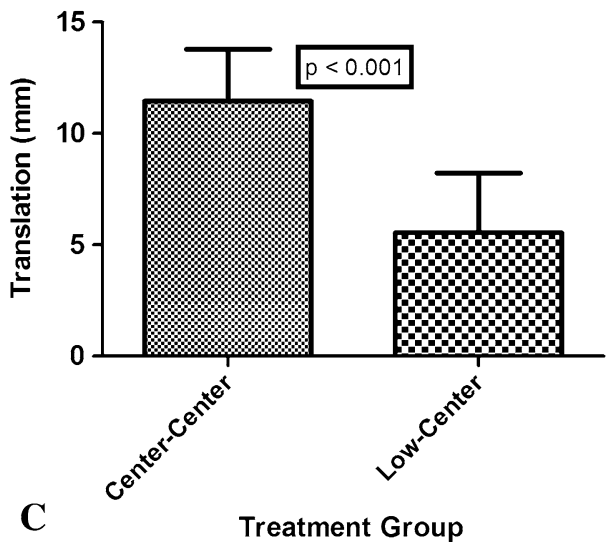

Fig. 4A-C The magnitude of fracture translation, defined as the distance between the Optotrak ${ }^{\circledR}$ femoral neck flag and distal femoral shaft flag, was significantly higher for (A) mean axial translation

pairs of samples needed for 0.8 power). There was no difference between low-center (1774 \pm 1016 cycles) and center-center $(1908 \pm 869$ cycles) treatment groups for the total number of cycles until cutout $(\mathrm{p}=0.588,1-\beta=0.05$, 224 pairs of samples needed for 0.8 power).

Fracture Kinematics

The magnitude of fracture translation at failure, defined as the distance between the Optotrak ${ }^{\circledR}$ femoral neck flag and distal femoral shaft flag, was greater in the center-center group $(20 \pm 2.8 \mathrm{~mm})$ compared with the low-center group $(15 \pm 3.4 \mathrm{~mm})$ at the time of failure $(\mathrm{p}=0.004)$ (Fig. $4 \mathrm{~A})$. Samples treated in the center-center group $(13 \pm 2.8 \mathrm{~mm})$ had significantly more fracture gap distraction, defined as distance between the femoral neck flag and proximal femoral shaft flag, compared with the low-center group $(7 \pm 4 \mathrm{~mm}$, $\mathrm{p}<0.001$ ) (Fig. 4B). Similarly, center-center treated samples

\section{Axial Fracture Distraction}

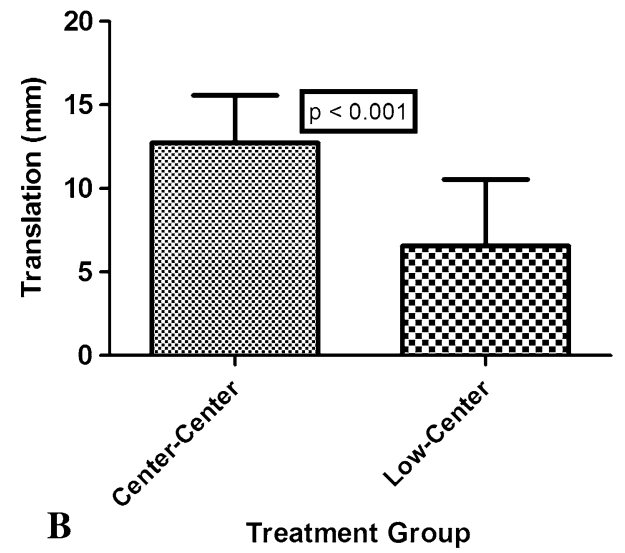

( $\mathrm{p}=0.004),(\mathbf{B})$ axial fracture distraction $(\mathrm{p}<0.001)$, and $(\mathbf{C})$ shear fracture translation $(\mathrm{p}<0.001)$.

$(12 \pm 2.3 \mathrm{~mm})$ had more shear fracture gap translation, defined as the distance between the proximal femoral shaft flag and distal femoral shaft flag compared with the low-center samples $(6 \pm 2.7 \mathrm{~mm}, \mathrm{p}<0.001)$ (Fig. 4C). The mean resultant translation and distraction translation were greater in the center-center group compared with the low-center group (Fig. 5) throughout the dynamic testing procedure.

\section{Discussion}

Fixation failure with lag screw cutout from the femoral head is a catastrophic complication after surgical fixation with intramedullary nailing. Multiple factors including fracture pattern, bone quality, patient age, quality of reduction, type of implant used, and position of the lag screw have been related to mechanism of failure $[1,4,13]$. We aimed to improve our understanding of optimal placement of lag screws as a predictive measure of implant 


\section{Fracture Translation Magnitude |M|}

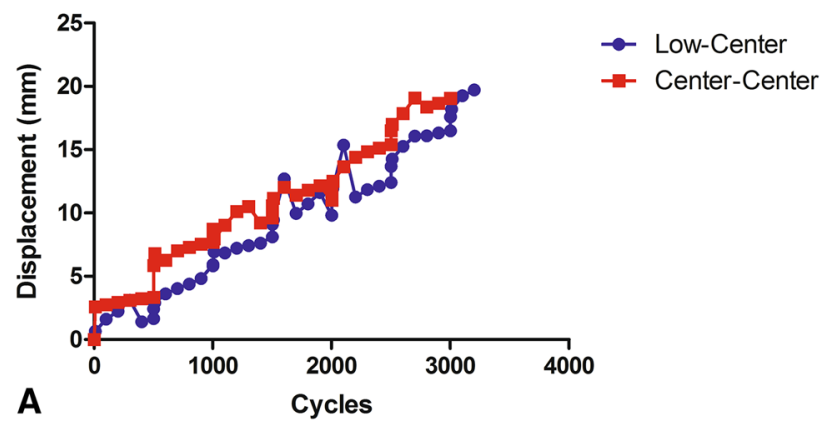

Fracture Distraction

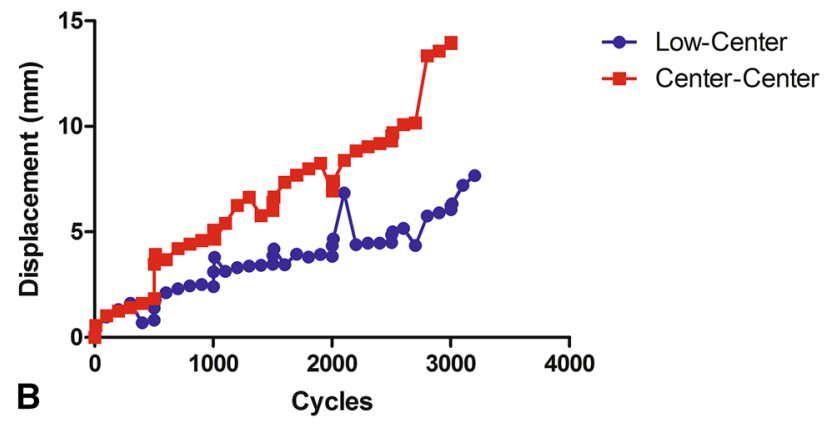

Fig. 5A-B Plots of (A) the mean fracture translation for the centercenter and low-center groups throughout cyclic loading and (B) the mean distraction translation between the two treatment groups throughout cyclic loading are shown.

and fracture stability in an unstable intertrochanteric fracture cadaveric model stabilized with a dynamically locked cephalomedullary nail. Our first goal was to examine if there was a difference between the center-center and low-center groups in cycles to failure and failure load. We found no statistically significant difference. Additionally, we analyzed fracture translation between the study groups during loading. Significantly, we found higher fracture translation in the center-center group versus the low-center group.

Our study has some limitations. We used fresh frozen cadaver tissue and consequently cannot foretell in vivo biomechanical properties. Additionally, by defining biomechanical failure as $1.5 \mathrm{~cm}$ of height loss, it was necessary to evaluate total deformation at different failure loads between specimens. However, failure at different loads is expected given that our cadavers had different bone density. Our samples were loaded in a vertical manner. One could argue that torsional forces should have been included in our evaluation to more closely mirror forces experienced by a patient treated with a dynamically locked intramedullary nail for an unstable intertrochanteric fracture. However, our objective was to preliminarily investigate biomechanical nail constructs and to this avail, we believe we have succeeded. Additionally it has been suggested that ideal lag screw position may be determined only after investigating various configurations of lag screw position relative to the coronal (AP x-ray) and the sagittal planes (lateral $\mathrm{x}$-ray) [10]. We recognize this as a limitation of our study and believe additional investigation examining AP position of the lag screw would be a valuable addition to the literature.

Baumgaertner et al. [1] introduced the concept of tip apex distance as a measure for being predictive of fixation failure by lag screw cutout. In their study, no complications of lag screw cutout were observed in any patient who had a tip apex distance less than $25 \mathrm{~mm}$. However, in patients with a tip apex distance greater than $30 \mathrm{~mm}, 27 \%$ of their patients experienced lag screw cutout. They concluded that tip apex distance greater than $30 \mathrm{~mm}$ increased the risk of cutout and should be avoided [1]. However, not all patients with greater than 30-mm tip apex distance experience cutout, thus additional factors need to be analyzed. In our testing, we evaluated various mechanical parameters of the stability of center-center lag screw orientation (mean tip apex distance of $21 \mathrm{~mm}$ ) versus low-center lag screwinstrumented samples (mean tip apex distance of $31 \mathrm{~mm}$ ). In our failure load and dynamic survival analyses, we found no difference between the center-center and lowcenter groups. With both constructs providing similar failure points in our biomechanical testing, we believe our data would suggest similar clinical results in patients treated with low-center lag screw fixation construct, despite possibly having a tip apex distance greater than $25 \mathrm{~mm}$.

To further understand the stability of the intramedullary nail fracture construct, we analyzed the 3-D motion of the construct during loading. During testing, at the time of failure, there was more fracture translation, fracture gap distraction, and shear fracture gap translation in the centercenter group (Fig. 4A). Thus, we think it is reasonable to infer from evaluation of the kinematics data that the lowcenter construct may provide a more stable fracture fixation construct than the center-center group. Some authors have examined the placement of lag screws and their correlation with lag screw cutout in the clinical setting. The highest rates of cutout reported by Baumgaertner et al. [1] occurred in the posteroinferior and anterosuperior zones. Consequently, they recommended central and deep insertion of the lag screw [1]. De Bruijn et al. [2], in their clinical series, found that tip apex distance should be minimized and the lag screw should be placed center-center or lowcenter. Kaufer et al. [6] argued for placement of the lag screw in the posteroinferior quadrant of the femoral head believing this area of the head and neck provided the best bone for screw placement because it represents the decussation of tension and compression trabeculae, thus improving proximal fragment control. These various studies provide effective clinical data; however, to our 
knowledge, no study to date has examined the strength of variable lag screw placement through vigorous biomechanical testing with cephalomedullary intramedullary nails in human cadaver bone.

Optimal lag screw position and resultant tip apex distance is debatable [11, 14, 15, 18]. In our biomechanical testing the low-center position with a tip apex distance greater than $25 \mathrm{~mm}$ showed equal if not superior stability as the centercenter construct with the currently accepted optimal tip apex distance less than $25 \mathrm{~mm}$. We now aim for a lag screw position that is low in the head on the AP radiograph and center on the lateral radiograph. Additional study is needed and we have begun a clinical study to further examine this topic as we recognize that no biomechanical testing can foretell in vivo loading and outcomes. From a biomechanical testing standpoint, additional studies examining different loading protocols, such as a torsional model, would be beneficial. Additionally, it is known that bone density throughout the neck is not uniform as the femoral head and neck have evolved to maximize strength while being subjected to various compressive and tensile forces [5]. It is our hypothesis that this variable quality of bone through which the lag screw is placed affects lag screw stability and consequently affects lag screw cutout. This is obviously an area in which we believe further investigation is warranted.

As evidenced by our testing, the low-center construct proved to be at least as stable as the center-center group although the tip apex distance was greater than $25 \mathrm{~mm}$ in our biomechanical loading model. These findings challenge previously accepted principles of optimal lag screw placement.

Acknowledgments The research was supported by [RIH Orthopaedic Foundation] and the National Institutes of Health [P20GM104937 (COBRE Bioengineering Core)].

\section{References}

1. Baumgaertner MR, Curtin SL, Lindskog DM, Keggi JM. The value of the tip-apex distance in predicting failure of fixation of peritrochanteric fractures of the hip. J Bone Joint Surg Am. 1995;77:1058-1064.

2. De Bruijn K, den Hartog D, Tuinebreijer W, Roukema G. Reliability of predictors for screw cutout in intertrochanteric hip fractures. J Bone Joint Surg Am. 2012;94:1266-1272.
3. Haynes RC, Poll RG, Miles AW, Weston RB. An experimental study of the failure modes of the Gamma Locking Nail and AO Dynamic Hip Screw under static loading: a cadaveric study. Med Eng Phys. 1997;19:446-453.

4. Haynes RC, Poll RG, Miles AW, Weston RB. Failure of femoral head fixation: a cadaveric analysis of lag screw cut-out with the gamma locking nail and AO dynamic hip screw. Injury. 1997;28:337-341.

5. Jang IG, Kim IY. Computational study of Wolff's law with trabecular architecture in the human proximal femur using topology optimization. J Biomech. 2008;41:2353-2361.

6. Kaufer H, Matthews LS, Sonstegard D. Stable fixation of intertrochanteric fractures. J Bone Joint Surg Am. 1974;56:899-907.

7. Kim WY, Han CH, Park JI, Kim JY. Failure of intertrochanteric fracture fixation with a dynamic hip screw in relation to preoperative fracture stability and osteoporosis. Int Orthop. 2001;25:360-362.

8. Kouvidis GK, Sommers MB, Giannoudis PV, Katonis PG, Bottlang M. Comparison of migration behavior between single and dual lag screw implants for intertrochanteric fracture fixation. J Orthop Surg Res. 2009;4:16.

9. Kubiak EN, Bong M, Park SS, Kummer F, Egol K, Koval KJ. Intramedullary fixation of unstable intertrochanteric hip fractures: one or two lag screws. J Orthop Trauma. 2004;18:12-17.

10. Kuzyk PR, Zdero R, Shah S, Olsen M, Waddell JP, Schemitsch EH. Femoral head lag screw position for cephalomedullary nails: a biomechanical analysis. J Orthop Trauma. 2012; 26:414-421.

11. Laskin RS, Gruber MA, Zimmerman AJ. Intertrochanteric fractures of the hip in the elderly: a retrospective analysis of 236 cases. Clin Orthop Relat Res. 1979;141:188-195.

12. 12 Lenz M, Perren SM, Gueorguiev B, Richards RG, Hofmann GO, Fernandez dell'Oca A, Hontzsch D, Windolf M. A biomechanical study on proximal plate fixation techniques in periprosthetic femur fractures. Injury. 2014; 45(suppl 1):S71-75.

13. Lorich DG, Geller DS, Nielson JH. Osteoporotic pertrochanteric hip fractures: management and current controversies. Instr Course Lect. 2004;53:441-454.

14. Parker MJ. Trochanteric hip fractures: fixation failure commoner with femoral medialization, a comparison of 101 cases. Acta Orthop Scand. 1996;67:329-332.

15. Simpson AH, Varty K, Dodd CA. Sliding hip screws: modes of failure. Injury. 1989;20:227-231.

16. Strauss E, Frank J, Lee J, Kummer FJ, Tejwani N. Helical blade versus sliding hip screw for treatment of unstable intertrochanteric hip fractures: a biomechanical evaluation. Injury. 2006;37:984-989.

17. Topp T, Muller T, Huss S, Kann PH, Weihe E, Ruchholtz S, Zettl RP. Embalmed and fresh frozen human bones in orthopedic cadaveric studies: which bone is authentic and feasible? Acta Orthop. 2012;83:543-547.

18. Wu CC, Shih CH, Lee MY, Tai CL. Biomechanical analysis of location of lag screw of a dynamic hip screw in treatment of unstable intertrochanteric fracture. J Trauma. 1996;41:699-702. 